\title{
Effects and Mechanisms of Bone Marrow Mesenchymal Stem Cell Transplantation for Treatment of Ischemic Stroke in Hypertensive Rats
}

\author{
Yulin Liu ${ }^{1, *}$, Ying Zhao ${ }^{2,3, *}$, Yu Min ${ }^{1}$, Kaifeng Guo ${ }^{1}$, Yuling Chen ${ }^{1}$, Zhen Huang ${ }^{1}$, Cheng Long ${ }^{2,3}$ \\ ${ }^{1}$ Department of Rehabilitation Medicine, Panyu Central Hospital, Guangzhou, China \\ ${ }^{2}$ South China Normal University-Panyu Central Hospital Foint Laboratory of Translational Medical Research, Panyu Central \\ Hospital, Guangzhou, China \\ ${ }^{3}$ School of Life Sciences, South China Normal University, Guangzhou, China
}

Background and Objectives: Stroke is the most common cause of human death and functional disability, resulting in more than 5 million deaths worldwide each year. Bone marrow mesenchymal stem cells (BMSCs) are a kind of stem cell that are able to self-renew and differentiate into many types of tissues. Therefore, BMSCs have the potential to replace damaged neurons and promote the reconstruction of nerve conduction pathways and connective tissue. However, it remains unknown whether transplanted BMSCs promote angiogenesis or improve the tissue microenvironment directly or indirectly through paracrine interactions. This study aimed to determine the therapeutic effect of BMSCs on ischemic stroke with hypertension in a rodent model and to explore the possible mechanisms underlying any benefits.

Methods and Results: Middle cerebral artery occlusion was used to establish the experimental stroke model. The area of cerebral infarction, expression of vascular endothelial growth factor (VEGF) and glial cell line-derived neurotrophic factor (GDNF), and increment of astrocyte were measured by TTC staining, western blot, real-time quantitative polymerase chain reaction (RT-qPCR) and immunocytochemistry. The results showed a smaller area of cerebral infarction and improved neurological function scores in animals treated with BMSCs compared to controls. The results of RT-qPCR and western blot assays showed higher expression of VEGF and GDNF in BMSC-treated animals compared with controls. Our study also showed that one round of BMSCs transplantation significantly promoted the proliferation of subventricular zone and cortical cells, especially astrocytes, on the ischemic side following cerebral ischemia. Conclusions: Above findings support that BMSCs have therapeutic effects for ischemic stroke complicated with hypertension, which may occur via up-regulated expression of VEGF and GDNF and reduction of neuronal apoptosis, thereby promoting the recovery of nerve function.

Keywords: Bone marrow mesenchymal stem cells, Ischemia, Stroke, Hypertensive

Received: August 2, 2021, Revised: September 13, 2021, Accepted: September 24, 2021, Published online: December 31, 2021

Correspondence to Zhen Huang

Department of Rehabilitation Medicine, Panyu Central Hospital, Guangzhou 511400, China

Tel: +86-020-34859320, Fax: +86-020-34859320, E-mail: doctor cdl@126.com

Co-Correspondence to Cheng Long

School of Life Sciences, South China Normal University, Guangzhou 510631, China

Tel: +86-020-85211372, Fax: +86-020-85211372, E-mail: longcheng@m.scnu.edu.cn

${ }^{*}$ These authors contributed equally to this work.

두 This is an open-access article distributed under the terms of the Creative Commons Attribution Non-Commercial License (http://creativecommons.org/licenses/by-nc/4.0/), which permits unrestricted non-commercial use, distribution, and reproduction in any medium, provided the original work is properly cited.

Copyright (c) 2022 by the Korean Society for Stem Cell Research 


\section{Introduction}

Stroke is one of the leading causes of death and disability worldwide, second only after ischemic heart disease (1). Stroke has become the leading cause of premature death and disease burden. About $94 \%$ of stroke burden is caused by controllable risk factors, such as high blood pressure, blood sugar, cholesterol level, smoking, body mass index, diet, and physical activity. Of these risk factors, hypertension is the most suitable for intervention. Acute treatment of ischemic stroke aims to restore perfusion of brain tissue and can be achieved with thrombolytic drugs or intravascular therapy. However, only a small proportion of stroke patients actually receive acute treatment. Thrombolytic therapy within 4.5 hours of onset is the most effective treatment for acute ischemic stroke (2). Studies have shown that approximately $25 \%$ of patients with ischemic stroke are eligible for thrombolytic therapy and $10 \sim 12 \%$ are eligible for intravascular treatment. Without therapeutic intervention, the ischemic core may show time-dependent expansion into the peri-infarct area, leading to additional cell death within days or even weeks after extended infarction and stroke (3, 4). For optimal treatment effects, tissue plasminogen activator (TPA) and thrombolytic therapy must be started within $3 \sim 6$ hours of stroke onset; the longer delay, the worse the effect will be. Although treatment within the effective window can reduce mortality after ischemic stroke, more than $60 \%$ of patients with cerebral apoplexy are left with varying degrees of movement, cognitive, language, and swallowing dysfunction (5). These consequences can greatly damage self-care ability, reducing quality of life for the individual and increasing the burden on their family and society. Therefore, more effective treatments for stroke are urgently needed.

Administration of bone marrow stem cells is a promising new strategy for the treatment of nerve damage caused by hypertension and stroke. Previous studies have shown that bone marrow stem cells can promote the recovery of limb dysfunction in patients with ischemic stroke, as well as gradually reduce other dysfunctions (6-9). Stem cells were transplanted into the brain of patients without excessive growth or deterioration with no adverse reactions such as tumor or infection. A number of studies have shown that both autologous and allogeneic bone marrow stem cells are safe for clinical use. Specifically, clinical trials have demonstrated that bone marrow stem cell therapy for ischemic stroke is safe and significantly improves dysfunction $(10,11)$. It has been reported that the mechanism that underlies the benefits of bone marrow stem cell therapy for patients with ischemic stroke arises from multiple factors (12). Several studies have described the beneficial effects of bone marrow-derived mesenchymal stem cells (MSCs) in the treatment of animal models of ischemic stroke $(13,14)$. There have been more than 20 international clinical explorations and trials on the use of mesenchymal stem cells and their derivatives for the treatment of stroke $(15,16)$, of which 12 used bone marrow mesenchymal stem cells (BMSCs). Together, these studies support the innate advantages of bone marrow stem cells in the treatment of stroke. After an ischemic event, bone marrow stem cells may be involved in reparative processes. There is growing evidence that BMSCs may be a variant useful for new therapy (17-19). In a meta-analysis of stem cell therapy for stroke, patients in the stem cell therapy group scored significantly better than the control group in terms of limb sensory function, motor function, and ability to perform activities of daily life, and there were no serious adverse reactions. In clinical trials, computed tomography (CT) imaging has shown a significant reduction in the size of cerebral infarction in stroke patients. Although great progress has been made in animal and clinical research on bone marrow mesenchymal stem cell transplantation, the experimental subjects were mainly healthy young male animals, and clinical statistics found that the proportion of ischemic stroke patients with hypertension was as high as $70 \%$, and the incidence of poor prognosis was also significantly higher than that without hypertensive, and the higher the patient's blood pressure, the worse the long-term living ability, and the higher the fatality rate (20). Currently, the efficacy of stem cell transplantation in the treatment of ischemic stroke with hypertensive remains unclear. To address this research gap, the present study adopted an ischemic stroke model with hypertension to assess the therapeutic effect of BMSCs and explore the potential mechanism of action.

\section{Materials and Methods}

\section{Experimental animal groups}

A total of 81 male 10-week-old (250 270 g) spontaneously hypertensive rats (SHR) were purchased from Beijing Weitong Lihua Experimental Animal Technology Co., Ltd. SHR were maintained in an air-conditioned room with a $12: 12 \mathrm{~h}$ light/dark cycle where the relative humidity was $60 \pm 10 \%$ and temperature was $22 \pm 2^{\circ} \mathrm{C}$. Water and food were freely available. All protocols and procedures involving animals were approved by the South China 
Normal University Animal Care and Use Committee.

The animals were randomly divided into the following three groups: (i) group A (sham group): normal SHR rats treated with a sham operation and then injected with $1 \mathrm{ml}$ phosphate-buffered saline (PBS) via the tail vein; (ii) group B (model group): SHR rats that successfully underwent cerebral artery occlusive modeling and were then injected with $1 \mathrm{ml}$ PBS via the tail veil; and Group C (BMSCs group): SHR rats that successfully underwent cerebral artery occlusive modeling and were then injected with $1 \times 10^{6}$ Bone marrow mesenchymal stem cells (BMSCs) via the tail vein.

\section{Neurological functional tests}

Neurological function was assessed using the modified neurological severity scores (mNSS) (see Table 1, Supplemental Digital Content, Supplemental Methods and Results, https:// www.docin.com/p-1005910882.html). The various groups are scored at 1, 3, 7, 14 days, respectively, each score repeated three times, then taking the average.

\section{Separation and identification of BMSCs}

Bone marrow samples from the femur and tibia of five male Wistar rats $(100 \sim 120 \mathrm{~g})$ were taken and inoculated on growth medium. The cells adhering to the bottom of the petri dish were used as the first generation of BMSCs and passaged several times for isolation. The second generation BMSCs were collected and identified by flow cytometry. For the BMSCs, CD90, CD34, CD45, CD44, CD11b/c, and CD29 were 92.35\%, 0.63\%, 0.61\%, 83.29\%, $0.35 \%$, and $88.78 \%$, respectively.

\section{Establishment of middle cerebral artery occlusion model}

Middle cerebral artery occlusion (MCAO) (21) was performed to establish the experimental stroke model. The MCAO procedure was performed on rats $(n=81)$ under anesthesia with chloral hydrate through reversible right middle cerebral artery occlusion for 90 minutes followed by reperfusion. During surgery and ischemia, the body temperature and head temperature were controlled at $37 \pm 0.5^{\circ} \mathrm{C}$ using a heating blanket and a heating lamp. A thread plug was inserted into the right internal carotid artery through the external carotid artery. Occlusion was confirmed and the filament was fixed in place for occlusion for 90 minutes. Rats were sacrificed at 3, 7 and 14 days after reperfusion to prepare a variety of tissue sections.

\section{Measuring the area of cerebral infarction}

At 3, 7, and 14 days after reperfusion, take out the whole brain of the rat. The prepared brain tissue was soaked in 2,3,5-triphenyte-trazoliumchloride (TTC) solution and incubated at a constant temperature of $37^{\circ} \mathrm{C}$ in the dark until the normal area and infarct area were dyed. The stained sections were placed in $4 \%$ formaldehyde solution for $24 \mathrm{~h}$, and the brain slices and filter paper were then removed. For each group, the cerebral infarction area (the percentage of cerebral infarction area to the total brain area) was analyzed and calculated using ImageJ software.

\section{Detection of VEGF and GDNF protein expression by western blot}

The brain samples from each group were homogenized and the total proteins from the homogenates were extracted using RIPA lysis buffer (Beyotime). Total protein concentrations were determined using the BCA protein concentration determination kit. Equal amounts of protein (30 $\mu \mathrm{g})$ were separated by $8 \%$ sodium salt (SDS)-Polyacrylamide gel electrophoresis (SDS-PAGE) and the separated proteins were then transferred onto a polyvinylidene difluoride membrane (Millipore, Bedford, MA, USA). Next, the membrane was blocked with 5\% skim milk at room temperature for $1 \mathrm{~h}$, followed by incubation with primary occluding antibody (1:1,000 dilution) at $4{ }^{\circ} \mathrm{C}$ overnight. After three washes with TBST (T: Tris; B: Buffer; S: Solution; $\mathrm{T}$ : Tween), the membrane was incubated with horseradish peroxidase-conjugated secondary antibody at room temperature for $1 \mathrm{~h}$. The targeted proteins were visualized using the enhanced chemiluminescence (ECL) substrate. The film was scanned and archived. Photoshop was used to sort and decolorize the images. The optical density of the target band was analyzed using the Alpha Software processing system. The level of $\beta$-actin protein served as an internal control.

\section{Assessment of VEGF and GDNF expression by real-time quantitative polymerase chain reaction (RT-qPCR)}

The RNA of the brain tissue for each group was extracted using TRIzol method and its cDNA was obtained by reverse transcription. The expression of VEGF and GDNF in brain tissue of each group was detected by RT-qPCR. Primer information: upstream of VEGF 5'-GCACTGGACCCTGGCTT TACT-3';Downstream of VEGF 5'-AACTTCACCACTTCA TGGGCTTT-3'; upstream of GDNF 5'-CAGTGACTCCAAT ATGCCCGA-3'; Downstream of GDNF5'-CGCTTGTTTATCTG GTGACCTTTT-3'; upstream of $\beta$-actin 5'-CTGGAGAAACC TGCCAAGTATG-3'; Downstream of $\beta$-actin 5'-GGTGGA AGAATGGGAGTTGCT-3'; initial denaturation at $95^{\circ} \mathrm{C}$ for $10 \mathrm{~min}$ followed by $95^{\circ} \mathrm{C}$ for $15 \mathrm{~s}, 60^{\circ} \mathrm{C}$ for $30 \mathrm{~s}$ and $65^{\circ} \mathrm{C}$ for $15 \mathrm{~s}$ to ensure 30 cycles. Samples were repeatedly 
measured 3 times, Calculating $2^{-\Delta \Delta \mathrm{Ct}}$.

\section{Calculation of astrocyte increment}

Astrocytes were measured after reperfusion for $24 \mathrm{~h}$. Endogenous neural stem cells were labeled with $\operatorname{BrdU}(50$ $\mathrm{mg} / \mathrm{kg}$, total injection 3, each rat injected three times, injection once every three hours). Put the brain in $4 \%$ of PFA, paraffin embedding coronary artery and cut, dye intermediate frequency of cortex, respectively. The cortex and SVZ were observed and the number of positive cells per field of view was calculated for each rat. The following values were also calculated: BrdU positive cells number, at the same time, record the immunofluorescence double labeling BrdU/GFAP, the number of cells to calculate astrocytes. We mainly observed new migration and differentiation of cells. In the lateral cortex and SVZ area of ischemia, counting BrdU/GFAP positive cells and BrdU positive cells, then calculate BrdU/GFAP positive cell per-

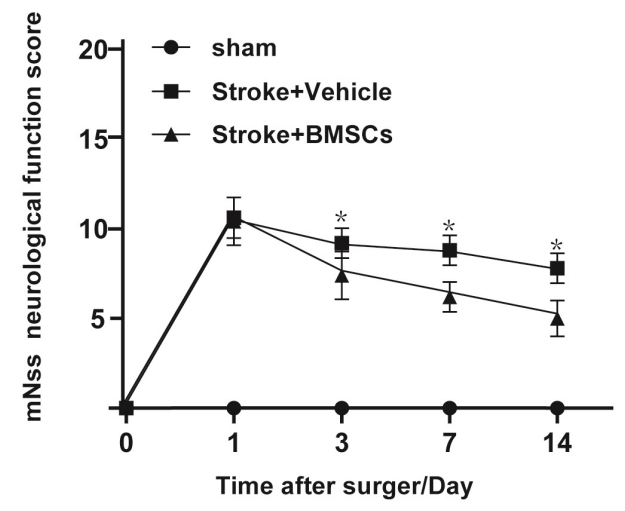

Fig. 1. Neurobehavioral scores of rats in each group $\left({ }^{*} p<0.05\right.$ vs Stroke+ Vehicle). mNSS test were assessed on day 1, 3, 7, and 14 after surgery respectively. The testing score of BMSC-treated rat was significantly better than the score of vehicle-treated stroke mice. ${ }^{*} \mathrm{p}<0.05$ (mNSS: modified Neurological Severity Scores). centage of the total number of BrdU positive cells.

\section{Statistical analysis}

All data were processed and analyzed using SPSS 20.0 software (IBM). Measurement data are reported as the mean \pm standard deviation (SD). Comparisons among more than two groups analyzed with one way analysis of variance (ANOVA) and LSD method was used to analyze the difference in mNSS scores. Kruskal-Wallis H-test was used to verify the differences in infarct volume, the expression of GDNF and VEGF proteins and mRNA, the proliferation of newborn cells. Results with $\mathrm{p}<0.05$ were considered statistically significant.

\section{Results}

\section{Neurological function results}

mNSS scores were assessed on day 1, 3, 7, and 14 after surgery (Fig. 1). According to the scores, there was no obvious neurological dysfunction in the sham group, but there were different degrees of neurological dysfunction in the other two groups. At each time point, the model group and BMSCs group had higher mNSS scores. As shown in Fig. 1, there was no significant difference between the model group and the BMSCs group on day 1; however, on day 3, the score in the BMSCs group decreased significantly and remained lower until the end of the experiment. This result suggests that the intervention treatment using BMSCs improved neurological function in SHR.

\section{TTC staining results}

After the brain slices of each group were made, the area of cerebral infarction was measured by TTC staining. The areas of cerebral infarction in the sham group, model group, and BMSCs group are shown in Fig. 2A. The slices
A

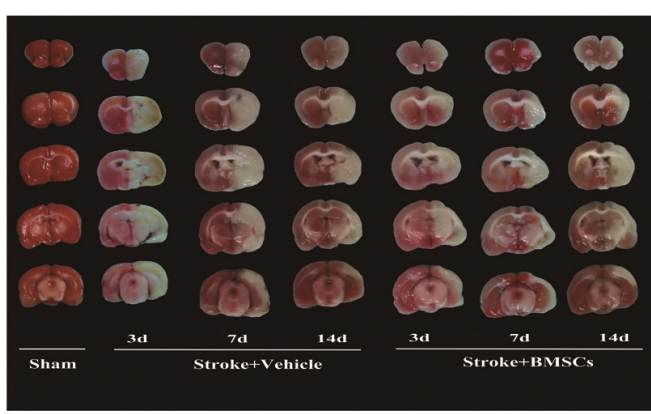

B

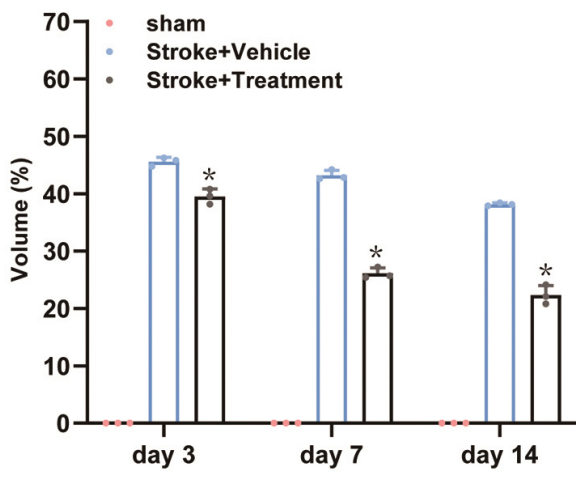

Fig. 2. BMSCs transplantation and neuroprotective effect after focal ischemia. (A) 2,3,5-Triphenyltetrazolium chloride (TTC) staining of coronal brain sections from vehicle-treated and BMSCs-treated barrel cortex stroke rat reveals the infarct area (white) in the cortex. (B) Quantified data show that the infarct volume (mean \pm SD) of BMSC-treated brains was significantly reduced compared with the vehicle-treated brain $\left({ }^{*} \mathrm{p}<0.05\right.$ vs Stroke+Vehicle) (TTC: 2,3,5-Triphenylttrazolium chloride). 
from the sham group are shown in red and there was no cerebral infarction. Compared with the sham group, the brain right side of the model group showed white cerebral infarction. The cerebral infarction area in the BMSCs group was significantly smaller than that in the model group (Fig. 2B). The results showed that the BMSCs intervention reduced the area of cerebral infarction on the ischemic side in hypertensive rats.

\section{Expression of VEGF and GDNF protein}

Western blotting was used to assess VEGF and GDNF protein expression levels (Fig. 3A). There was no significant change in expression in the sham group. However, in the BMSCs group, VEGF significantly increased $(\mathrm{p}<$ 0.05 ; Fig. $3 \mathrm{~B}$ and $3 \mathrm{C}$ ). Although the amount of the ex- pression of GDNF in the BMSCs group had no obvious change on days $3,7,14$, but compared with the sham group and model group there were significant difference $(p<0.05)$. This result suggests that transplantation of BMSCs significantly promoted the expression of VEGF and GDNF proteins in ischemic brain tissue.

\section{RT-qPCR results}

The expression of VEGF and GDNF messenger ribonucleic acid (mRNA) was detected by the real-time fluorescence quantitative polymerase chain reaction method. On days 3, 7, and 14, VEGF and GDNF mRNA were expressed in the sham group; however, there was no significant difference between the model group and the sham group. The expression of GDNF mRNA in the BMSCs
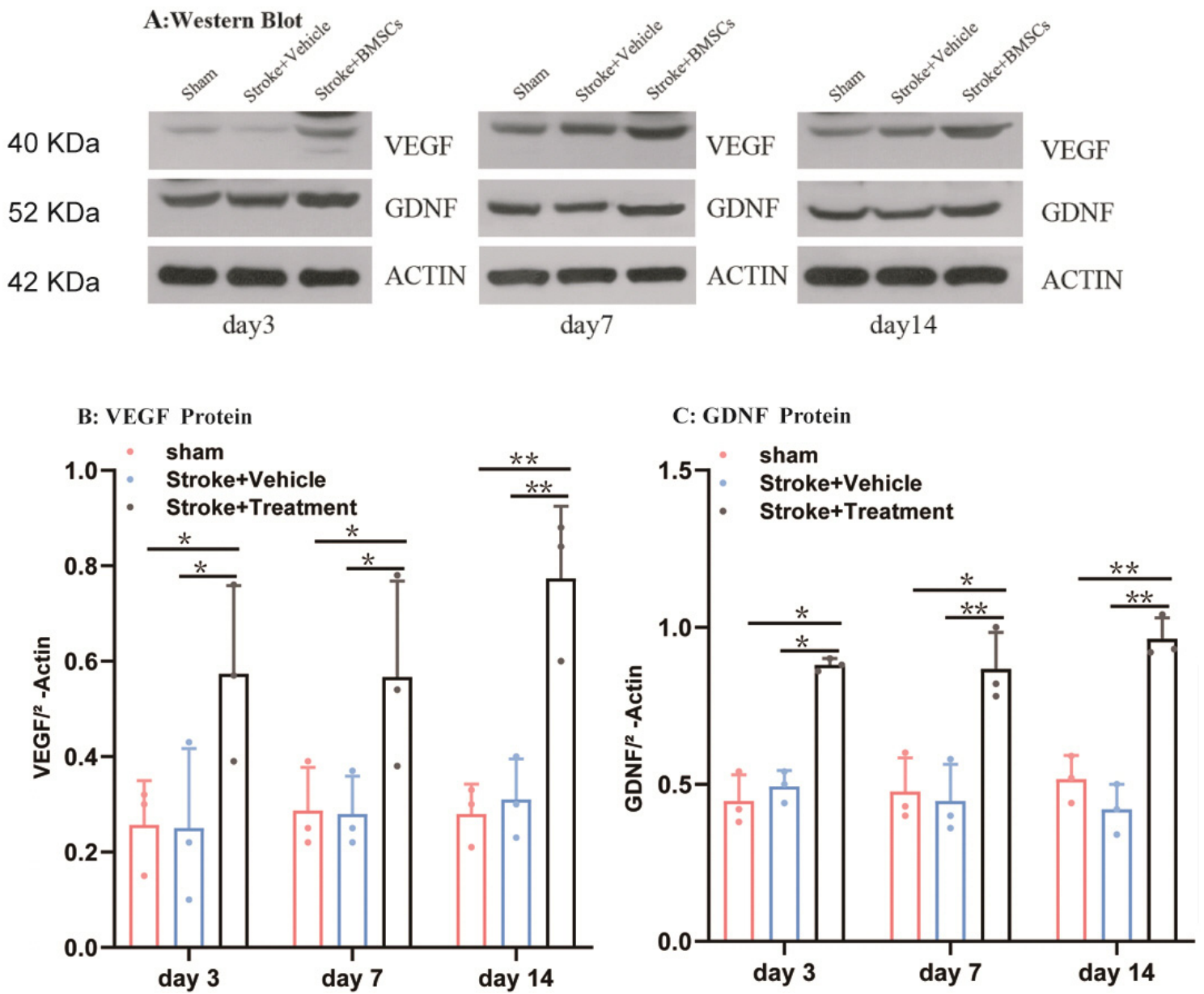

Fig. 3. Expression of GDNF and VEGF proteins in the peri-infarct cortex. The protein expression levels of GDNF and VEGF in each group were analyzed with Western blot on days 3, 7, and 14 respectively (A). Representative bands of growth associated GDNF and VEGF expression levels (B, C). Quantitative determination of immune proteins of GDNF and VEGF, GDNF and VEGF were quantitatively analyzed on days 3,7 and 14 in each group. Compared with the sham group and model group, BMSCs therapy significantly enhanced the expression of GDNF and VEGF $\left({ }^{*} p<0.05,{ }^{* *} p<0.01\right)$ (VEGF: vascular endothelial growth factor; GDNF: glial cell line-derived neurotrophic factor). 

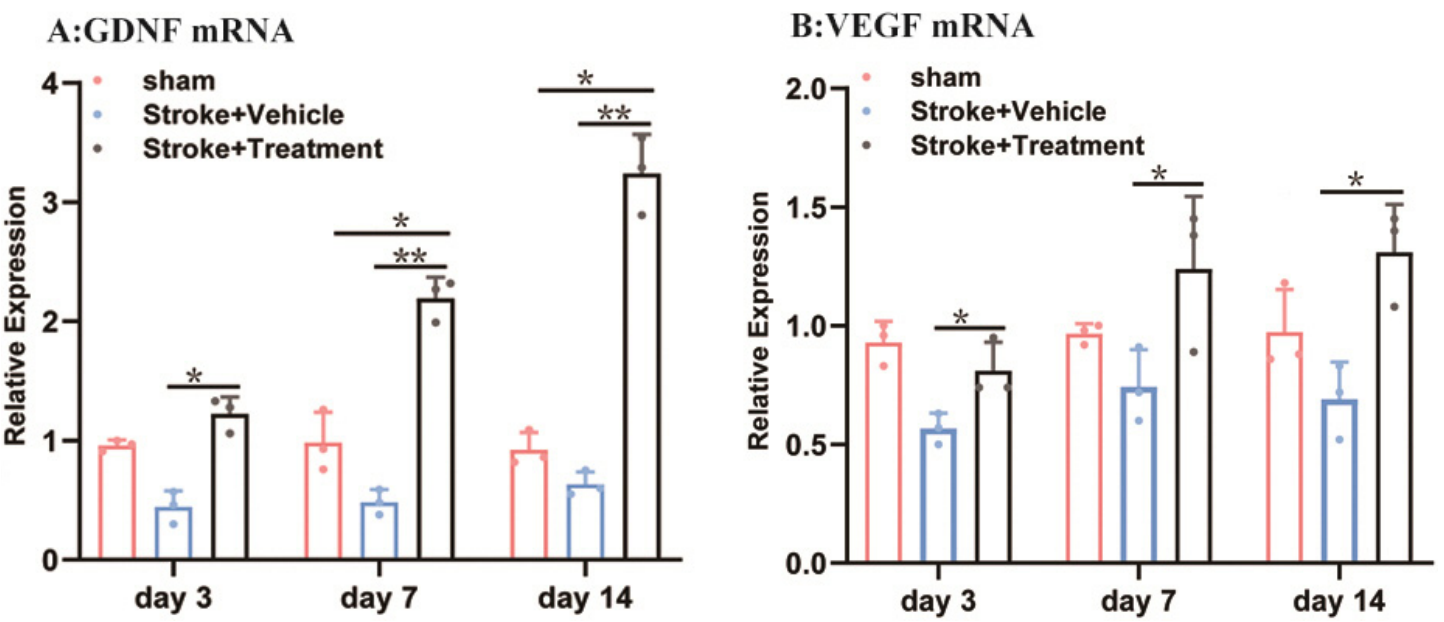

Fig. 4. Expression of GDNF and VEGF mRNA in the peri-infarct cortex. The mRNA expression levels of GDNF and VEGF were assessed by RT-PCR at different time periods (A, B). GDNF and VEGF were quantitatively analyzed on days 3, 7 and 14 in each group. Compared with the sham group and model group, BMSCs group significantly increased the expression of GDNF and VEGF $\left({ }^{*} p<0.05, * * p<0.01\right)$ (VEGF: vascular endothelial growth factor; GDNF: glial cell line-derived neurotrophic factor).

group was significantly higher than that in the sham group on days 7 and 14 (Fig. 4A), and it was also significantly higher than that in the model group on days 3,7 , and 14, and the expression reached a peak on day 14. The expression of VEGF mRNA in the BMSCs group was significantly higher than that in the model group on days 3, 7, and 14 (Fig. 4B).

\section{Post-treatment change in hippocampal astrocytes}

The results of immunofluorescence staining showed that, on days 3, 7 and 14, there was a small number of new cells in the model group compared with the sham group and a large number of new cells could be seen in the ischemic side SVZ and cortex of the BMSCs group (Fig. 5A D). The number of BrdU-positive cells in the BMSCs group at each time point was significantly higher than that in the model group $(p<0.05)$ and the sham group $(\mathrm{p}<0.05)$. For the SVZ and cortex, the proliferation of astrocytes in the model group was not significantly different from that in the sham group on days 3, 7, and 14 . There was a significant difference in the proliferation of astrocytes on the ischemic side in the BMSCs group (Fig. $5 \mathrm{E} \sim \mathrm{H})$. These results suggest that BMSC transplantation significantly promoted the proliferation of SVZ and cortical cells, especially astrocytes, on the ischemic side after cerebral ischemia.

\section{Discussion}

Cerebrovascular obstruction causes nerve cells ischemic hypoxia, which may lead to disability. Although the blood vessels are once again smooth after a series of medical measures, secondary reperfusion injuries may increase the original nerve damage and potentially aggravate the degree of disability. Treatment of thrombolytic and vascular thrombus is the most common clinical application (22), but the consequences are secondary ischemia-reperfusion injury, which can further aggravate the condition. BMSCs have emerged as a therapeutic candidate for a variety of diseases including stroke (23). These multipotent cells are easily isolated from bone marrow and rapidly expand in vitro (24). They are safe and effective for repair and reconstruction of brain tissue, and of bone tissue (25), and may participate in anti-inflammatory, anti-apoptosis, and regulating autophagy. Previous studies have demonstrated that disruption of the blood-brain-barrier (BBB) plays an important role in the development of neurological dysfunction after stroke $(26,27)$. In previous studies, transplantation of BMSCs was found to reduce BBB permeability, as shown by a low level of Evans blue penetration (28). So that the transplanted BMSCs can cross the BBB and repair the damage.

There are many lateral circles in ischemic semi-dark belt, there are still a large number of half-dead neurons, and if it can quickly restore ischemic semi-dark blood flow in a short time, the brain cells can be resurrected and recovered, which can be obvious reducing infarction area and improving patient prognosis. The recovery of ischemic semi-dark blood flow is closely related to the formation of new blood vessels. VEGF has become the strongest angiogenic factor since 1983 was discovered. BMSCs can enhance angiogenesis and neuron regeneration in the cere- 
A

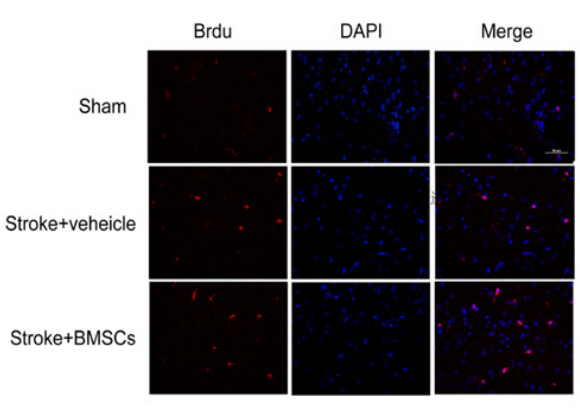

C

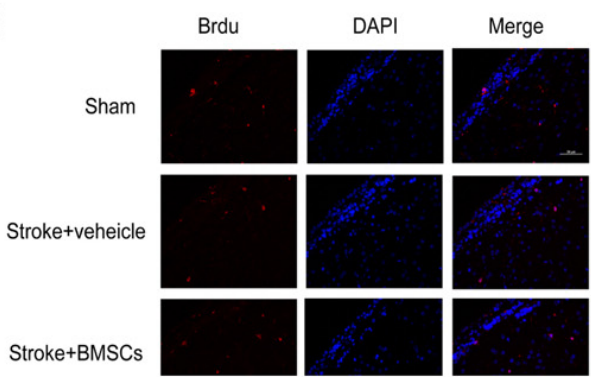

$\mathrm{E}$

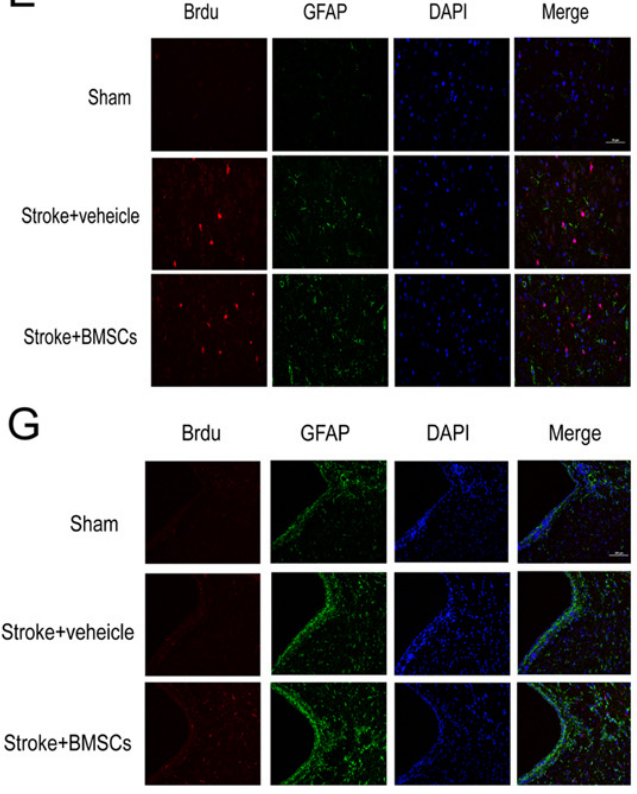

B

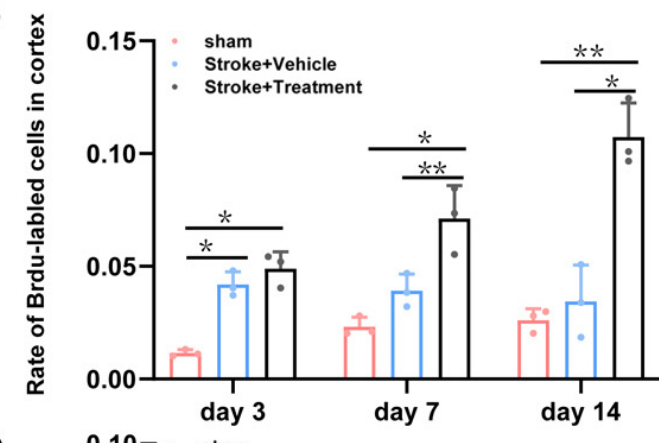

D

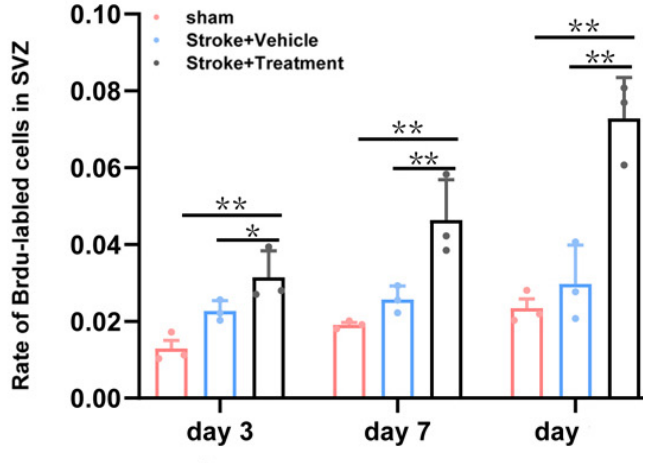

F

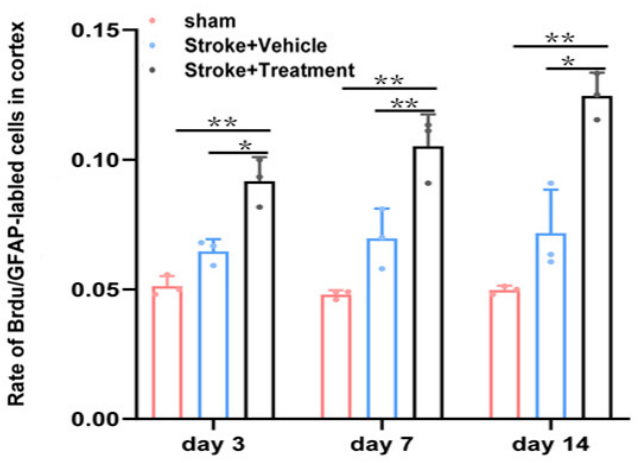

$\mathrm{H}$

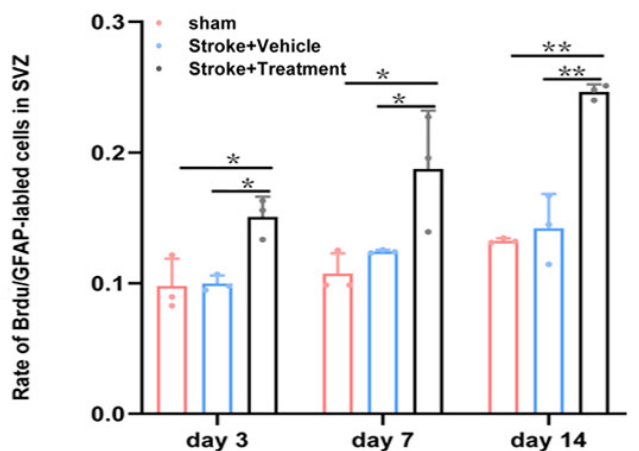

Fig. 5. Proliferation rate of newborn cells in cortex area of ischemic side of rats in each group and at different time points, the method of immunofluorescence staining was used. (A, B) The proliferation of newborn cells was detected in the SVZ area of the ischemic side of rats. Compared with the model group and the sham group, the cell proliferation rate of the BMSCs group was significantly higher than that of the model group and the control group in SVZ area. (C, D) The proliferation of newborn cells was detected in the cortex area of the ischemic side of rats. Compared with the model group and the sham group, the cell proliferation rate of the BMSCs group was significantly higher than that of the model group and the control group in cortex area. (E, F) Proliferation rate of GFAP in SVZ area of ischemic side of rats in each group and at different time points. Compared with the model group and the sham group, the increment rate of astrocytes of the BMSCs group was significantly higher than that of the model group and the control group in SVZ area. (G, H) Proliferation rate of GFAP in cortex area of ischemic side of rats in each group and at different time points. Compared with the model group and the sham group, the increment rate of astrocytes of the BMSCs group was significantly higher than that of the model group and the control group in cortex area $(* \mathrm{p}<0.05, * * \mathrm{p}<0.01)$ (SVZ: Subventricular Zone). 
bral ischemic area and surrounding areas.

In this study, the cerebral infarction area in BMSCs group was significantly smaller than that of the model group, and neurological deficit scores also decreased significantly, indicating that BMSCs have a protective effect on ischemic stroke in hypertension rats, which is consistent with previous research (29). We also found that the expression of the protein and mRNA of VEGF in BMSCS group were significantly higher than that of the model group, indicating that BMSCS can reduce the infarction area by enhanced angiogenesis around ischemia, which in turn promotes the recovery of neurological function. Glial cell-derived neurotrophic factor was the most important neurotrophic which can promote motor nerve development and regeneration. LHSHEN et al found that on day 8 after cerebral infarction, the transplantation of BMSCs significantly increased GDNF expression, promoted the proliferation and migration of neuroblasts in SVZ, and reduced apoptosis of ischemia-half dark belt cells. Our studies have also found that after transplantation BMSCs, the protein and mRNA expression of GDNF in cerebral ischemia regions have increased significantly higher than model groups and blank groups, which may be BMSCS to activate local astrocytes in semi-dark zone. The latter releases a lot of GDNF, which promotes the improvement of the function.

The initial astrocytes were considered to be "glue in the brain", providing the structural bracket necessary for neuronal function, but now a large amount of evidence shows that the interaction of star-colored cells and neurons far exceeds a simple structure Auxiliary concept. The connection between neurons is synaptic, although healthy brain neurons can remain intact in their own life, but they are constantly formed, enhanced, reduced, or dead. Astrocytes play a key role in determining the exact generation of new synapses, thereby affecting the microenvironment of the ischemic zone to improve the effect of improving neural kinetic energy. After transplantation of BMSCs in this study, the proliferation ratio of astrocytes was significantly increased with the model group and the sham group, which may be the formation of new synapses, by affecting the microenvironment of ischemic zone to promote functional improvement. In summary, BMSCs can migrate and survive in cerebral ischemia, creating microenvironment facilitating survival and regenerative microenvironment to repair injuries, reducing infarction area, and effectively improving nerve function.

Although BMSCs cannot directly convert into neurons, more and more evidence show that stem cell intervention can increase the degree of expansion of astrocytes, and re- pair the entire neural circuit by affecting the astrocytes. The results of this study point in the same direction as those of Song \& Mohamad who reported that transplantation of BMSCs improved neurological dysfunction and up-regulated the expression of neurotrophic factors and related growth protein, ultimately achieving the role of protecting the ischemic side of the brain (30). Uniquely, this study also used an animal model with hypertension, which is more in line with clinical needs. Compared with normal rats, the model of cerebral ischemia in SHR rats can lead to more severe neurological impairment. As a result, in previous studies, the efficacy of stem cells in healthy animal models may be exaggerated. Thus, further research involving disease model animals can provide a new treatment scheme for solving the thorny problem of dysfunction of ischemic stroke in patients with hypertension.

In conclusion, increasing research evidence supports that stem cell transplantation can treat ischemic stroke and improve damage to neurological function. In the present study, it was found that BMSC transplantation improved defects in spontaneously hypertensive ischemic stroke model rats by enhancing secretion of VEGF and GDNF, preventing neuronal injury and death, improving the pathological state of neurons, promoting the repair and regeneration of injured neurons, and enhancing the proliferation and differentiation of endogenous neural stem cells. These findings suggest that BMSCs have a therapeutic effect in spontaneously hypertensive stroke rats. Despite many studies on BMSC transplantation, this treatment is not widely used in clinics, indicating that the conversion between basic and clinical research has not been successful. More basic experimental data are needed to support this transformation. Stem cells show great potential for regeneration. Future research should continue to use these cells for cellular and gene therapy applications, explore additional therapeutic mechanisms, and combine multiple mechanisms to achieve the best therapy. Ultimately, the aim is to generate highly functional mesenchymal stem cells for cell-based clinical applications.

\section{Acknowledgments}

This work has been financially supported by Panyu District Science and Technology Planning Project (No: 2018-Z04-07). The Experimental Center of the School of Life Sciences of South China Normal University provided the facilities for this study.

\section{Potential Conflict of Interest}

The authors have no conflicting financial interest. 


\section{References}

1. Camen S, Haeusler KG, Schnabel RB. Cardiac imaging after ischemic stroke or transient ischemic attack. Curr Neurol Neurosci Rep 2020;20:36

2. Churilov L, Fridriksdottir A, Keshtkaran M, Mosley I, Flitman A, Dewey HM. Decision support in pre-hospital stroke care operations: a case of using simulation to improve eligibility of acute stroke patients for thrombolysis treatment. Comput Oper Res 2013;40:2208-2218

3. Fisher M, Garcia JH. Evolving stroke and the ischemic penumbra. Neurology 1996;47:884-888

4. Xie W, Zhao ZH, Yang QM, Wei FH. The efficacy of the seamless transfer of care model to apply for the patients with cerebral apoplexy in China. Int J Nurs Sci 2015;2:5257

5. Chen J, Li Y, Katakowski M, Chen X, Wang L, Lu D, Lu M, Gautam SC, Chopp M. Intravenous bone marrow stromal cell therapy reduces apoptosis and promotes endogenous cell proliferation after stroke in female rat. J Neurosci Res 2003;73:778-786

6. Chopp M, Li Y. Treatment of neural injury with marrow stromal cells. Lancet Neurol 2002;1:92-100

7. De Keyser J. Autologous mesenchymal stem cell transplantation in stroke patients. Ann Neurol 2005;58:653-654; author reply 654-655

8. Li M, Cui Z, Niu Y, Liu B, Fan W, Yu D, Deng J. Synaptogenesis in the developing mouse visual cortex. Brain Res Bull 2010;81:107-113

9. Li Y, Chen J, Chen XG, Wang L, Gautam SC, Xu YX, Katakowski M, Zhang LJ, Lu M, Janakiraman N, Chopp M. Human marrow stromal cell therapy for stroke in rat: neurotrophins and functional recovery. Neurology 2002;59: 514-523

10. Cízková D, Rosocha J, Vanický I, Jergová S, Cízek M. Transplants of human mesenchymal stem cells improve functional recovery after spinal cord injury in the rat. Cell Mol Neurobiol 2006;26:1167-1180

11. Badr AE, Yin W, Mychaskiw G, Zhang JH. Dual effect of $\mathrm{HBO}$ on cerebral infarction in MCAO rats. Am J Physiol Regul Integr Comp Physiol 2001;280:R766-R770

12. Li WY, Choi YJ, Lee PH, Huh K, Kang YM, Kim HS, Ahn YH, Lee G, Bang OY. Mesenchymal stem cells for ischemic stroke: changes in effects after ex vivo culturing. Cell Transplant 2008;17:1045-1059

13. Ullrich E, Bosch J, Aigner M, Völkl S, Dudziak D, Spriewald B, Schuler G, Andreesen R, Mackensen A. Advances in cellular therapy: 5th International Symposium on the clinical use of cellular products, 19 and 20 March 2009, Nürnberg, Germany. Cancer Immunol Immunother 2010;59:1745-1756

14. Baksh D, Song L, Tuan RS. Adult mesenchymal stem cells: characterization, differentiation, and application in cell and gene therapy. J Cell Mol Med 2004;8:301-316

15. Jeong H, Yim HW, Cho YS, Kim YI, Jeong SN, Kim HB, $\mathrm{Oh}$ IH. Efficacy and safety of stem cell therapies for pa- tients with stroke: a systematic review and single arm meta-analysis. Int J Stem Cells 2014;7:63-69

16. Yu H, Xu Z, Qu G, Wang H, Lin L, Li X, Xie X, Lei Y, He X, Chen Y, Li Y. Hypoxic preconditioning enhances the efficacy of mesenchymal stem cells-derived conditioned medium in switching microglia toward anti-inflammatory polarization in ischemia/reperfusion. Cell Mol Neurobiol 2021;41:505-524

17. Dzau V, Mirotsou M, inventor; Duke University, assignee. Stem cell derived paracrine factor $\mathrm{hl}$ for use in reducing cell death in cardiac tissue. EP2548569B1. 2014 Jan 29.

18. Zhang LL, Zhang HT, Cai YQ, Han YJ, Yao F, Yuan ZH, Wu BY. Anti-inflammatory effect of mesenchymal stromal cell transplantation and quercetin treatment in a rat model of experimental cerebral ischemia. Cell Mol Neurobiol 2016;36:1023-1034

19. Deng QJ, Xu XF, Ren J. Correction to: effects of SDF-1/ CXCR4 on the repair of traumatic brain injury in rats by mediating bone marrow derived mesenchymal stem cells. Cell Mol Neurobiol 2021;41:617-618

20. Bejan-Angoulvant T, Saadatian-Elahi M, Wright JM, Schron EB, Lindholm LH, Fagard R, Staessen JA, Gueyffier F. Treatment of hypertension in patients 80 years and older: the lower the better? A meta-analysis of randomized controlled trials. J Hypertens 2010;28:1366-1372

21. Fang J, Tang XM, Wang S, Zheng B, Li ZX, Sun XH, Wu J, Fang J, Lu Q, Dong YR, Zhai Y, Zhang X, Pan H, Liu JR. Efficacy and safety of combined therapy of intravenous thrombolysis and endovascular thrombectomy in patients with acute ischemic stroke. Chin J Clin Neurosci 2015;23: 481-487

22. Nejadnik H. Bone marrow derived mesenchymal stem cell (BM MSC) application in articular cartilage repair $[\mathrm{PhD}$ dissertation]. Singapore: National University of Singapore; 2013.

23. Seo JH, Cho SR. Neurorestoration induced by mesenchymal stem cells: potential therapeutic mechanisms for clinical trials. Yonsei Med J 2012;53:1059-1067

24. Sekiya I, Larson BL, Smith JR, Pochampally R, Cui JG, Prockop DJ. Expansion of human adult stem cells from bone marrow stroma: conditions that maximize the yields of early progenitors and evaluate their quality. Stem Cells 2002;20:530-541

25. Lei H, Lei L, Dong A, Hong T, Zhang Y, Zhou H, Song C, Mou C. Transplantation of bone marrow mesenchymal stem cells in the brain of rats with cerebral ischemia-reperfusion injury decreases expression of TNF- $\alpha$ and increases expression of TGF $\beta 1$. Chin J Histochem Cytochem 2017;26:327-335

26. Yang Y, Rosenberg GA. Blood-brain barrier breakdown in acute and chronic cerebrovascular disease. Stroke 2011;42: 3323-3328

27. Keep RF, Zhou N, Xiang J, Andjelkovic AV, Hua Y, Xi G. Vascular disruption and blood-brain barrier dysfunction in intracerebral hemorrhage. Fluids Barriers CNS 2014;11: 18 
28. Wang C, Fei Y, Xu C, Zhao Y, Pan Y. Bone marrow mesenchymal stem cells ameliorate neurological deficits and blood-brain barrier dysfunction after intracerebral hemorrhage in spontaneously hypertensive rats. Int J Clin Exp Pathol 2015;8:4715-4724

29. Liu Z, Li Y, Zhang RL, Cui Y, Chopp M. Bone marrow stromal cells promote skilled motor recovery and enhance contralesional axonal connections after ischemic stroke in adult mice. Stroke 2011;42:740-744

30. Song M, Mohamad O, Gu X, Wei L, Yu SP. Restoration of intracortical and thalamocortical circuits after transplantation of bone marrow mesenchymal stem cells into the ischemic brain of mice. Cell Transplant 2013;22:2001-2015 